\title{
Research on vibration noise of the composite laminated plate structure based on the FEM-BEM
}

\author{
Yang Xu, Dongyan Shi, Xiaoyan Teng and Hongxi Ren \\ Harbin Engineering University, Harbin, P. R. China
}

\begin{abstract}
Based on the FEM-BEM hybrid method, the effects of the angle of composite layer, number of layers and the opening position of a composite material and opening form are studied in order to get these three aspects' influence law on the vibration and acoustic radiation properties of the composite laminated plate structure, which can provide some reference for the engineers to design the composite laminated plate structure.
\end{abstract}

\section{Introduction}

The composite laminated plate structure is widely used in the fields of navigation, vehicle engineering, aerospace and other important engineering fields due to its excellent performance. Zhao Fei et al. theoretical calculated the natural frequencies and modes of the composite laminated plates based on the finite element method and the layered theory, and then through the experimental verification, the displacement mode of the laminated plates is solved by the layered finite element model, so as to solve the natural frequency[1]. Based on the semianalytical method of the theory of state vector equation, a new numerical simulation method was proposed by Guanghui Qingden et al. to analyze the free vibration properties of the reinforced laminated plates [2]. U.N. Band, Y.M. Desai presents a unique finite element method for the analysis of the static and free vibration properties of thick plates and sheets. This method combines the $3 \mathrm{D}$ hybrid layered and the equivalent monolayer theory [3]. X.W. Yin et al. studied the acoustic radiation law of the concrete double-period parallel reinforced laminated plates under the point loading and the infinite fluid loading by the theoretical method [4]. Cao Xiongtao gives the five kinds of plateshell structure' vibration and acoustic radiation prediction method, and the five structures includes the two groups of reinforced laminated plates, the ring rib reinforcement of constrained damping laminated cylindrical shells, the multi-periodic laminated cylindrical shells and so on[5].

Due to the characteristics of the composite structure, their mechanical properties not only depends on the form of the structure, but also on the mechanical properties of the each single-layer materials that form the macroscopic structure and the relationship between the layers. After a lot of paper-researching about the related published literature, it is not difficult to find out that most of the researchers has confined to focus on the research of form of the composite material structure, the theoretical modeling and the experimental study on the performance of related materials, etc. Therefore, the angle of composite layer, number of layers and the opening position of a composite material and opening form have great significance to the effect of the structure vibration noise. This paper is based on the FEM-BEM hybrid method, the effects of the angle of composite layer, number of layers and the opening position of a composite material and opening form are studied in order to get these three aspects' influence law on the vibration and acoustic radiation properties of the composite laminated plate structure, which can provide some reference for the engineers to design the composite laminated plate structure.

\section{FEM-BEM analysis model}

Figure 1 shows the flow chart of the structural analysis model based on the FEM-BEM hybrid method in this paper. It can be divided into the following three steps:

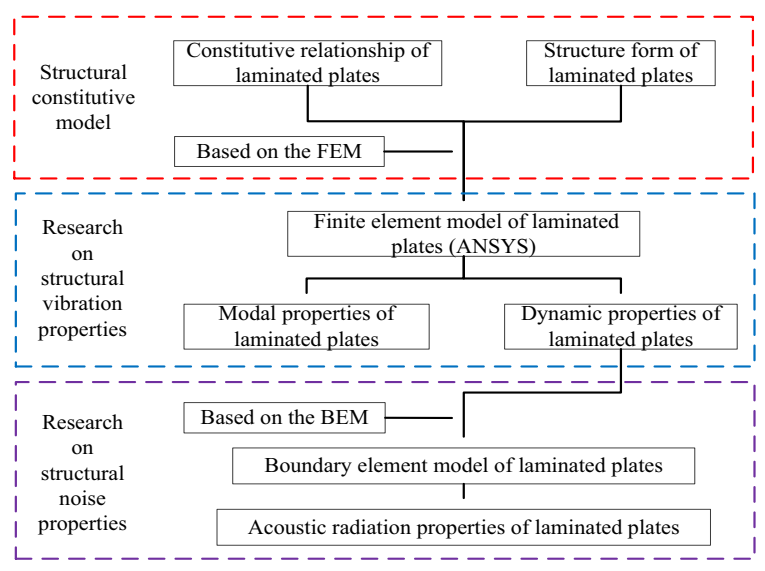

Figure 1. The flow chart of the structural analysis model. 


\subsection{Classical laminated plate theory}

The difference between the composite laminated structure and the homogeneous material structure is that the laminated structure has macroscopic heterogeneity in the thickness, so the analysis of the laminated plates needs to be based on each single layer material. Only clearing the stiffness properties of each single layer material can obtain the overall stiffness properties of the laminated plate structure. Understanding the strength properties of each single layer can determine the overall strength properties of the laminated plates. Therefore, the macroscopic mechanical analysis of single layer materials is the basis of the laminated structure analysis. In classical theory, the Kichhoof hypothesis of the plate and the Kirchhoff-Love hypothesis of the shell are proposed [6]. The laminated structure of composite material is mostly orthotropic materials, the stress-strain relationship of the orthotropic materials will be described below on the basis of the Kichhoof hypothesis. The unidirectional composite material is not generally used alone, but is used as a base unit in a laminated plate, in combination with other materials. And the Structural width to thickness ratio is generally large in the practical engineering applications. Therefore, the mechanical properties of the single layer composite materials can be regarded as the plane stress problem, that is:

$$
\sigma_{3}=\tau_{23}=\tau_{31}=0
$$

The relationship of the stress and strain in the main direction of the material are as follows:

$$
\left[\begin{array}{l}
\varepsilon_{1} \\
\varepsilon_{2} \\
\gamma_{12}
\end{array}\right]=\left[\begin{array}{ccc}
S_{11} & S_{12} & 0 \\
S_{12} & S_{22} & 0 \\
0 & 0 & S_{66}
\end{array}\right]\left[\begin{array}{l}
\sigma_{1} \\
\sigma_{2} \\
\tau_{12}
\end{array}\right]
$$

Where the $S_{i j}$ is the compliance coefficient, their relationship with the engineering elastic constants are as follows:

$$
S_{11}=\frac{1}{E_{1}}, S_{22}=\frac{1}{E_{2}}, S_{66}=\frac{1}{G_{12}}, S_{12}=-\frac{\mu_{12}}{E_{1}}=-\frac{\mu_{21}}{E_{2}}
$$

Where the $\mu_{12}$ is the ratio of the strain $\left(-\varepsilon_{2} / \varepsilon_{1}\right)$ caused by the individual strain $\sigma_{1}, \mu_{21}$ is the ratio of the strain $\left(-\varepsilon_{1} / \varepsilon_{2}\right)$ caused by the individual strain $\sigma_{2}$.

From equation (3), the elastic constants of the orthotropic thin plate includes: $E_{1}, E_{2}, G_{12}, \mu_{12}$ and $\mu_{21}$. From the reciprocal theorem,

$$
\frac{\mu_{12}}{E_{1}}=\frac{\mu_{21}}{E_{2}}
$$

So there are four independent elastic constants. Inverse the equation (2), the constitutive equation of the composite laminated plates can be obtained:

$$
\left[\begin{array}{l}
\sigma_{1} \\
\sigma_{2} \\
\tau_{12}
\end{array}\right]=\left[\begin{array}{ccc}
Q_{11} & Q_{12} & 0 \\
Q_{12} & Q_{22} & 0 \\
0 & 0 & Q_{66}
\end{array}\right]\left[\begin{array}{l}
\varepsilon_{1} \\
\varepsilon_{2} \\
\gamma_{12}
\end{array}\right]
$$

Where the $Q_{i j}$ is the reduced stiffnesses, their relationship with the engineering elastic constants are as follows:

$$
Q_{11}=\frac{E_{1}}{1-\mu_{12} \mu_{21}}, Q_{22}=\frac{E_{2}}{1-\mu_{12} \mu_{21}}, Q_{66}=G_{12}, Q_{12}=\mu_{12} Q_{11}
$$

On the basis of the constitutive equation, the corresponding finite element analysis model will be established, and reasonable material and geometric parameters will be set according to the constitutive equation.

\subsection{Establishment of the structure finite element analysis model based on the FEM}

The mechanical foundation of finite element analysis is the elastic mechanics, and the solution principle of the equation is the weighted residual method or the functional extreme value principle. The realization of the method is the numerical discrete technology, and the technical carrier is the finite element analysis software. In this paper, ANSYS software will be used for analysis. First, a finite element model is needed. According to the geometry of the laminated plates, the thickness ratio of the laminated plates is more than 10, so the SHELL99 element is used in ANSYS software to establish the finite model [7]. In order to be closer to the actual engineering application, this paper selects the T300/BMP316 (the reinforcement is the carbon fiber T300, the Substrate is the BMP316), which is commonly used in engineering, to establish the model and the following analysis of the vibration acoustic radiation properties. The properties of T300 / BMP316 laminated plates and the geometrical parameters used in this paper are shown in Table 1 and Table 2.

Table 1. T300 / BMP316 material performance parameters.

$E_{1}=128.8 \mathrm{GPa}, E_{2}=E_{3}=8.3 \mathrm{GPa}, G_{12}=G_{13}=G_{23}=4.1 \mathrm{GPa}$
$\mu_{12}=\mu_{13}=\mu_{23}=0.355, \rho=1578 \mathrm{~kg} / \mathrm{m}^{3}$

Table 2. Lamination size parameters.

\begin{tabular}{|c|c|c|}
\hline Material & $\begin{array}{c}\text { Length } \mathrm{L} \times \\
\text { width } \mathrm{B}(\mathrm{mm})\end{array}$ & $\begin{array}{c}\text { Single layer thickness } \\
\times \text { number of layers } \\
(\mathrm{mm})\end{array}$ \\
\hline T300/BMP316 & $180 \times 48$ & $0.6 \times 3$ \\
\hline Layer angle $\left({ }^{\circ}\right)$ & Pore size & Opening position \\
\hline$[0 / \theta / 0]$ & 0.024 & $(90,24)$ \\
\hline
\end{tabular}

In the case of using different unit to grid, not all of the performance singular need to be clear during the definition of material performance parameters In the process of modeling. For example: the SHELL99 element is used in this paper, as this unit is shell element, the thickness ratio is generally large. Therefore, the normal tensile modulus $G_{23}$ of the material does not affect the 
natural frequency of the laminated plates, that is to say, the value of $G_{23}$ can be arbitrary under the premise that the software can calculate. At the same time, the values of Poisson's ratio $\mu_{13}$ and $\mu_{23}$ can be omitted and the software will default to 0 , which does not affect the modal analysis of the laminated plates. Finally, the finite element model of the structure is obtained by dividing the grid, as shown in Figure 2.

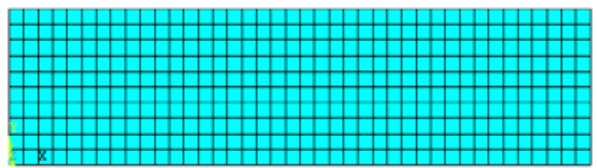

Finite element model of non - damage laminated plate.

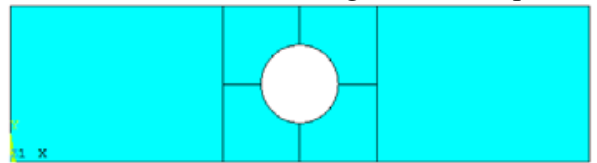

Split for the laminated plate model easy to mesh.

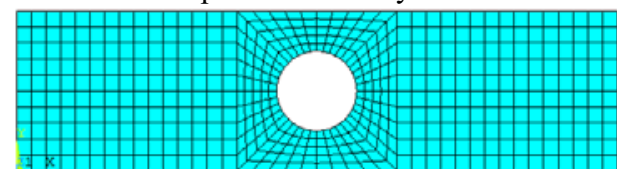

A round hole laminated plate model with good mesh. L1

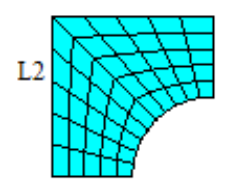

A 1/4 area mesh model around the round hole.

Figure 2. The finite element analysis model in this paper.

\subsection{Establishment of the structure finite element analysis model based on the BEM}

Boundary element method is a numerical calculation method for structural acoustic wave analysis, this method integrates the differential equation into boundary integral equation, and then discretizes the boundary into finite elements, and obtains the equations only with the unknown nodes on the boundary to solve the problem [8]. In this paper, the vibration response of the structure under the harmonic excitation is analyzed, and the acoustic simulation is also carried out. Under the action of the harmonic excitation, the acoustic pressure $\mathrm{p}$ generated by the structural vibration in the medium satisfies the Helmholtz equation:

$$
\nabla^{2} p+k^{2} p=0
$$

Where the $p$ is the sound pressure, $k$ is the number of the sound wave, $k=\omega / c, \omega$ is the excitation circular frequency, $c$ is the sound velocity in the medium, in this paper, $c=343 \mathrm{~m} / \mathrm{s}$. Structural vibration external field acoustic radiation problem belongs to the Neumman problem, that is to say, the surface normal velocity is given as $\partial p / \partial n=i \omega \rho v_{n}$, where the $\rho$ is the medium density, $v_{n}$ is the structural surface normal velocity. At the same time, the sound pressure must also satisfy the condition that the sound pressure is zero at infinity, using the Green's function can obtain the Helmholtz integral equation:

$$
C\left(\mathbf{r}_{a}\right) p\left(\mathbf{r}_{a}\right)=\iiint_{\Omega}\left[G\left(\mathbf{r}_{b}, \mathbf{r}_{a}\right) \frac{\partial p\left(\mathbf{r}_{b}\right)}{\partial n}-\frac{\partial G\left(\mathbf{r}_{b}, \mathbf{r}_{a}\right)}{\partial n} p\left(\mathbf{r}_{b}\right)\right] d \Omega\left(\mathbf{r}_{b}\right)
$$

Where the $\mathbf{r}_{b}$ is the point on the surface of radiation, $\mathbf{r}_{a}$ is the point in space.

$C\left(\mathbf{r}_{a}\right)$ is depending on the location of $\mathbf{r}_{a}$ in the sound field. For the smooth surface $\Omega$, when $\mathbf{r}_{a}$ is within the radiator, $C\left(\mathbf{r}_{a}\right)=0$; when $\mathbf{r}_{a}$ is the nonsingular point on the radiation surface, $C\left(\mathbf{r}_{a}\right)=2 \pi$; when $\mathbf{r}_{a}$ is in the radiation field, $C\left(\mathbf{r}_{a}\right)=4 \pi$.

The boundary element method is used to solve the Helmholtz equation. Boundary element method is divided into the direct boundary element method and the indirect boundary element method. The direct boundary element method requires that the acoustic grid be closed, and the mesh of the indirect boundary element method can be closed or not. The direct boundary element method needs to discretize the boundary $\Omega_{a}$ into a number of elements $\left(\Omega_{a e}\right)$ and nodes. The sound pressure $p$ and the normal velocity $v_{n}$ at any point within each element can be determined by the acoustic pressure $a_{p i}$, the normal velocity $a_{v i}$ on the node belonging to the element and the shape function $N_{i}^{e}$ of the element. In this paper, the normal velocity $a_{v i}$ is a known quantity obtained by the finite element method. Which is:

$$
\begin{gathered}
p\left(\mathbf{r}_{a}\right)=\sum_{i=1}^{n_{e}} N_{i}^{e}\left(\mathbf{r}_{a}\right) \cdot a_{p i}, \mathbf{r}_{a} \in \Omega_{a e} \\
v_{n}\left(\mathbf{r}_{a}\right)=\sum_{i=1}^{n_{e}} N_{i}^{e}\left(\mathbf{r}_{a}\right) \cdot a_{v i}, \mathbf{r}_{a} \in \Omega_{a e}
\end{gathered}
$$

Where the $n_{e}$ is the number of nodes on an element $\Omega_{a e}$.

The element shape function $N_{i}^{e}$ is 1 on the node $i$ and 0 on the other nodes of the element. The element shape function $N_{i}^{e}$ can be assembled into a global shape function $N_{i}$, which is 1 on the node $i$ and 0 on the other nodes. So on the entire boundary element mesh $\Omega_{a}$, there is:

$$
\begin{array}{r}
p\left(\mathbf{r}_{a}\right)=\sum_{i=1}^{n_{a}} N_{i} \cdot p_{i}=\mathbf{N}_{i}\left\{p_{i}\right\}, \mathbf{r}_{a} \in \Omega_{a} \\
v_{n}\left(\mathbf{r}_{a}\right)=\sum_{i=1}^{n_{a}} N_{i} \cdot v_{n i}=\mathbf{N}_{i}\left\{v_{n i}\right\}, \mathbf{r}_{a} \in \Omega_{a}
\end{array}
$$

Where the $n_{a}$ is the number of nodes for all boundary element mesh.

At present a lot of commercial software already have mature acoustic boundary element analysis method, this 
paper uses the Virtual.lab software to analyze the structure and obtain the acoustic radiation properties of the structure [9].

\section{Numerical structure analysis}

\subsection{The verification of the modal}

It's necessary to verify the correctness of the finite element analysis model established in this paper before the vibration noise analysis, and then gives a verification example, the material and structure parameters are shown in Table 3, where the $L$ and $B$ are the length and width of the plate, $H$ is the thickness of the plate, $\theta$ is the layer angle. The mesh size of the example is $80 \times 80 \times 6$, and respectively calculated with the SHELL99 and SOLID46 element, the results are shown in Table 4. In order to verify the correctness of the proposed method, the exact solution results [10] are also listed in Table 4. The Table 4 shows that the results from the FEM analysis model based on the finite element software ANSYS is in good agreement with the results of the literature. The calculation results of the SOLID46 element is more accurate, and the error of the SHELL99 element is relatively large, but the error is also controlled within $1 \%$, which shows the correctness of this method.

Table 3. The material parameters and geometrical dimensions of the example.

$E_{1}=15 E_{2}, E_{2}=E_{3}=10 \mathrm{GPa}, G_{12} / E_{2}=G_{13} / E_{2}=0.6, G_{23} / E_{2}=0.5$
$\mu_{12}=\mu_{13}=\mu_{23}=0.25, \rho=1450 \mathrm{~kg} / \mathrm{m}^{3}, L=B=1 \mathrm{~m}, H / B=0.01, \theta=30^{\circ}$

Table 4. Comparison of the calculation results.

\begin{tabular}{|c|c|c|c|c|c|}
\hline \multirow[b]{2}{*}{$\begin{array}{l}\text { Ord } \\
\text {-er }\end{array}$} & \multirow[b]{2}{*}{$\begin{array}{l}\text { Literature } \\
\text { results[10] } \\
\quad(\mathrm{Hz})\end{array}$} & \multicolumn{2}{|c|}{ SOLID46 } & \multicolumn{2}{|c|}{ SHELL99 } \\
\hline & & $\begin{array}{c}\text { Frequen } \\
-c y \\
(\mathrm{~Hz})\end{array}$ & $\begin{array}{c}\text { Error } \\
(\%)\end{array}$ & $\begin{array}{c}\text { Frequen } \\
\text {-cy } \\
(\mathrm{Hz})\end{array}$ & $\begin{array}{l}\text { Error } \\
(\%)\end{array}$ \\
\hline 1 & 9.45 & 9.4484 & 0.0169 & 9.3979 & 0.551 \\
\hline 2 & 21.28 & 21.279 & 0.0047 & 21.167 & 0.531 \\
\hline 3 & 46.06 & 46.064 & 0.00868 & 45.812 & 0.55 \\
\hline 4 & 60.73 & 60.722 & 0.0132 & 60.289 & 0.726 \\
\hline
\end{tabular}

\subsection{The vibration acoustic radiation properties analysis}

On the basis of validating the finite element analysis model, this paper will further study the vibration acoustic radiation properties of the composite laminated plates. In order to study the effects of the layer angle and the number of layers on the vibration acoustic radiation properties of the laminated plates, several models were established for comparison as shown in Table 5. The boundary condition of all the laminated plates are the one-end constraint, and the analysis process is shown in Figure 1. Firstly, the harmonic response of each plate is calculated and analyzed by the acoustic boundary element method to obtain the acoustic radiation curves of each plate, as shown in Figure 3. It can be seen from Figure 3 that the acoustic radiation properties of the structure will produce a maximum peak value of the sound pressure at the position corresponding to the natural frequency with the change of the excitation frequency, especially at the fundamental frequency position. After the fundamental frequency position, the increase of the sound pressure value is not particularly evident. In addition, it also can be seen from Figure 3 that the peak sound pressure of the structure is increased with the increase of the number of layers. When $\mathrm{m}$ exceeds 2 , the trend gradually tend to be stable. In order to study the effects of the layer angle and the number of layers on the vibration acoustic radiation properties of the laminated plates comprehensively, the dB-Frequency curves of different layer angle and the number of layers are combined to draw a threedimensional surface, as shown in Figure 4. For the angle of the layer, when the angle value is constant and observe the trend of the sound pressure $\mathrm{dB}$ with the change of frequency, we can see that all the curves obtain the peak sound pressure near the first and the third order natural frequency values, which shows that all the plates produce resonance near the first and the third order natural frequency values, and the noise generated thereby is maximized so that the sound pressure value $\mathrm{dB}$ is peaked. When the frequency is constant and observe the trend of the sound pressure $\mathrm{dB}$ with the change of the layer angle, we can see that when the frequency is not in the vicinity of the plate natural frequency, the sound pressure $\mathrm{dB}$ with the change of the layer angle is not obvious. For the number of layers, when the number of layers is 2 , the excitation frequency corresponding to the peak sound pressure has obvious differences with the other laminated plates. When the number of layers increases, the excitation frequency corresponding to the peak sound pressure is consistent.

Table 5. Model parameters of the different number of layers.

\begin{tabular}{|c|c|c|c|}
\hline Code & $\begin{array}{c}\text { Size } \\
(\mathrm{mm})\end{array}$ & $\begin{array}{c}\text { Single layer } \\
\text { thickness } \\
\times \text { number of } \\
\text { layers }\end{array}$ & Layer angle \\
\hline $\begin{array}{c}\mathrm{A}_{n}(n=0,1,2 \\
, \ldots, 18)\end{array}$ & $180 \times 48$ & $0.6 \times 3$ & {$[0 / 5 n / 0]$} \\
\hline $\begin{array}{c}\mathrm{B}_{m}(m=1,2,3 \\
, \ldots, 15)\end{array}$ & $180 \times 48$ & $(2 / 2 \mathrm{~m}) \times 2 \mathrm{~m}$ & $\begin{array}{c}{[0 / 90]_{m}(m=1,} \\
2,3, \ldots, 15)\end{array}$ \\
\hline
\end{tabular}

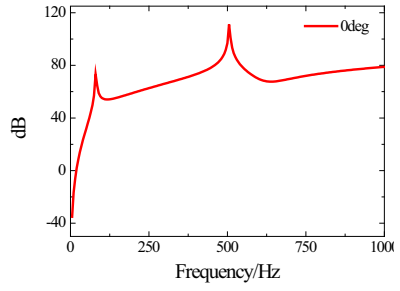

(a) Layer angle.

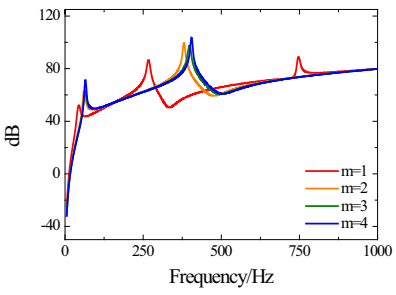

(b) Number of layers.
Figure 3. The vibration acoustic radiation curve of the structure.

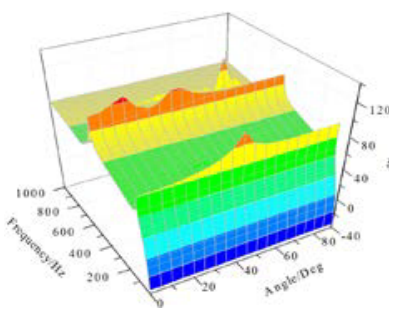

(a) Layer angle.

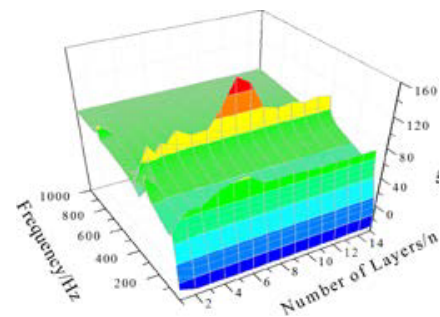

(b) Number of layers.
Figure 4. The sound pressure value of different layer angle and 
the number of layers curves with the change of the excitation frequency.

Furthermore, the effect of the opening structure on the vibration acoustic radiation of the structure is studied, the results are shown in Figure 5.

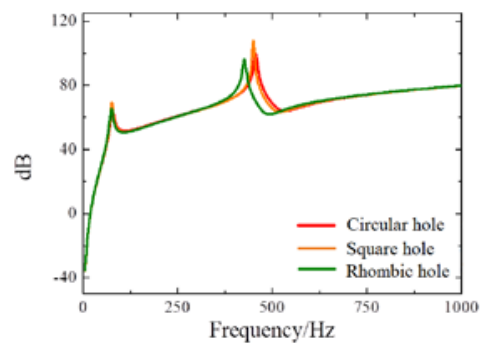

(a) The acoustic radiation curves of the laminated plates with different opening forms.

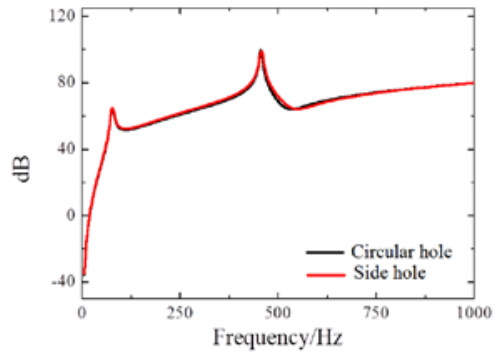

(b) The acoustic radiation curves of the laminated plates with different opening positions.

Figure 5. the acoustic radiation curves of the laminated plates with different opening.

The Figure 5 (a) shows that the third order natural frequency of the laminated plates with the rhombic hole is lower, so the excitation frequency corresponding to the peak sound pressure is smaller, and the second is the laminated plates with the square hole, the laminated plates with the circular hole is the largest. And the sound pressure of the laminated plates with the square hole at the natural frequency is the largest, the second is the laminated plates with the circular hole, and the laminated plates with the rhombic hole is the smallest. In Figure 5 (b), the excitation frequency corresponding to the peak sound pressure of the two curves are closed, which illustrate that the natural frequency of the two plates are closed. Among them, the sound pressure value of the curve of the laminated plates with the side hole is slightly larger than that of laminated plates with the circular hole between the first order natural frequency value and the third order natural frequency value, which indicates that the plate vibration can produce a larger noise caused by the side hole. In practical engineering, the influence cannot be neglected when there are many side holes.

\section{Conclusions}

Based on the FEM-BEM hybrid method, the effects of the angle of composite layer, number of layers and the opening position of a composite material and opening form are studied in this paper, and the influence law of these three aspects on the vibration acoustic radiation properties of the composite laminated plate structure are as follows:
(1) The vibration radiation noise of the composite laminated plate structure changes with the change of the angle, especially between the angle of 20 to 80 degree;

(2) For the number of layers, when the number of layers is 2 , the excitation frequency corresponding to the peak vibration radiation noise has obvious differences with the other laminated plate structure. When the number of layers increases, the excitation frequency corresponding to the peak sound pressure is consistent.

(3) The opening structure has little effect on the peak value of the vibration radiation noise of the composite laminated plates, only affecting its peak position. The excitation frequency corresponding to the peak sound pressure of the laminated plates with the rhombic hole is smaller, and the second is the laminated plates with the square hole, the laminated plates with the circular hole is the largest.

The above conclusions are only qualitative analysis of the results obtained. In the actual engineering applications, it's necessary to comprehensively consider the various factors so as to choose the reasonable angle of composite layer, number of layers, the opening form and so on.

\section{References}

1. Zhao F, Wu J W, Zhao L S. Analysis for Natural Frequencies and Mode Shapes of Laminated Composite Plates Using Layerwise Theory. Noise and Vibration Control, 34, 34-40 (2014)

2. Qing G, Qiu J, Liu Y. Free vibration analysis of stiffened laminated plates. International Journal of Solids and Structures, 43, 1357-1371 (2006)

3. Band U N, Desai Y M. Coupled higher order and mixed layerwise finite element based static and free vibration analyses of laminated plates. Composite Structures, 128, 406-414 (2015)

4. Yin X W, Gu X J, Cui H F, et al. Acoustic radiation from a laminated composite plate reinforced by doubly periodic parallel stiffeners. Journal of Sound and Vibration, 306, 877-889 (2007)

5. Cao X T. Vibrational and Acoustic Prediction Method of Laminated Plates and Shells (2007)

6. Zhang J, Zhao W Z, Zhang W Y. Research on acoustic-structure sensitivity basing on FEM and BEM. Journal of Vibration Engineering, 18, 366-370 (2005)

7. Wang X M, Li Y Q, Xu H W. ANSYS Structural Analysis Unit and Its Application, 200-300 (2011)

8. Tan J A. The Application of Boundary Element Methods in Environmental Acoustics. Noise and Vibration Control, 20-22 (2002)

9. Li Z G, Zhan F L. Virtual.Lab Acoustic-Acoustic Simulation Computing Advanced Application Examples. 105-160 (2010)

10. Qu Y, Yuan G, Wu S, et al. Three-dimensional elasticity solution for vibration analysis of composite rectangular parallelepipeds. European Journal of Mechanics - A/Solids, 42, 376-394 (2013) 